\title{
Role of mechanical strain-activated PI3K/Akt signaling pathway in pelvic organ prolapse
}

\author{
BING-SHU LI* , WEN-JUN GUO*, LI HONG, YAO-DAN LIU, \\ CHENG LIU, SHA-SHA HONG, DE-BIN WU and JIE MIN
}

\begin{abstract}
Department of Obstetrics and Gynaecology, Renmin Hospital of Wuhan University, Wuhan, Hubei 430060, P.R. China
\end{abstract}
Received July 11, 2015; Accepted April 27, 2016

DOI: $10.3892 / \mathrm{mmr} .2016 .5264$

\begin{abstract}
Mechanical loading on pelvic supports contributes to pelvic organ prolapse (POP). However, the underlying mechanisms remain to be elucidated. Our previous study identified that mechanical strain induced oxidative stress (OS) and promoted apoptosis and senescence in pelvic support fibroblasts. The aim of the present study is to investigate the molecular signaling pathway linking mechanical force with POP. Using a four-point bending device, human uterosacral ligament fibroblasts (hUSLF) were exposed to mechanical tensile strain at a frequency of $0.3 \mathrm{~Hz}$ and intensity of $5333 \mu \varepsilon$, in the presence or absence of LY294002. The applied mechanical strain on hUSLF resulted in apoptosis and senescence, and decreased expression of procollagen type I $\alpha 1$. Mechanical strain activated phosphatidylinositol-4,5-bisphosphate 3-kinase (PI3K)/Akt signaling and resulted in downregulated expression of glutathione peroxidase 1 and $\mathrm{Mn}$-superoxide dismutase, and accumulation of intracellular reactive oxygen species. These effects were blocked by administration of LY294002. Furthermore, it was demonstrated that PI3K/Akt was activated in the uterosacral ligaments of POP patients, and that OS was increased and collagen type I production reduced. The results from the present study suggest that mechanical strain promotes apoptosis and senescence, and reduces collagen type I production via activation of PI3K/Akt-mediated OS signaling pathway in hUSLF. This process may be involved in the pathogenesis of POP as it results in relaxation and dysfunction of pelvic supports.
\end{abstract}

Correspondence to: Professor Li Hong, Department of Obstetrics and Gynaecology, Renmin Hospital of Wuhan University, 238 Jiefang Road, Wuhan, Hubei 430060, P.R. China

E-mail: springtea@outlook.com

*Contributed equally

Key words: mechanical stretch, PI3K/Akt signaling pathway, oxidative stress, pelvic organ prolapse, human uterosacral ligament fibroblasts

\section{Introduction}

The female pelvic floor is subjected to a constantly changing mechanical load due to intra-abdominal pressure and gravity. Clinical studies have identified that vaginal labor and multi-gravidity are key risk factors for pelvic organ prolapse (POP) (1-3). External tensile load is associated with POP via its mechanical stretch and compression on pelvic support $(4,5)$. Pelvic supports are collagen-rich, dense connective tissues predominantly composed of fibroblasts, these allow the tissue to respond directly to the surrounding environment and they are particularly mechanoresponsive (6). Fibroblasts secrete extracellular matrix (ECM), maintain tissue homeostasis, and are involved in repair and remodeling. Previous studies have demonstrated that the biomechanical properties of pelvic supports are abnormally altered in POP patients (7-9), thus, it is hypothesized that mechanical force is associated with POP (5). Based on these findings, the present study hypothesizes that mechanical loading is a potential mechanism involved in POP. An increased understanding of the specific molecular signaling pathways associating physical force with POP may be beneficial in defining the underlying etiologies of the development of POP and aid in the development of novel treatment options for women with this disorder.

Mechanotransduction, including mechanical sensing molecules (for example, integrins) and formation of stress fibers and focal adhesions convert extracellular mechanical stimulations into intracellular signals, and result in a series of cellular responses. Transmembrane integrins transmit extracellular mechanical signals via intracellular kinase networks, and Akt is involved in the mechanotransduction as a component of these kinase networks (6,10-12). Akt, a serine/threonine kinase, is a downstream effector of integrin linked kinase, it is activated by mechanical strain and regulates a broad range of cellular functions $(13,14)$. When unstimulated, Akt is present in the cytoplasm, and two regulatory phosphorylated sites (Thr308 and Ser473) are unphosphorylated. Following biochemical or mechanical stimulation, Akt is recruited to the plasma membrane. Activated Akt phosphorylates its downstream targets, which translocate to subcellular locations (15). Mechanical strain activates the phosphatidylinositol-4,5-bisphosphate 3-kinase (PI3K)/Akt signaling pathway in order to regulate cellular apoptosis, proliferation, ageing and metabolism. It has been demonstrated in fibroblasts 
from the skin, osteoblasts, airway smooth muscle cells and periodontal ligament fibroblasts $(6,13,14,16)$, however, to the best of our knowledge, it has not yet been demonstrated in pelvic support fibroblasts (PSF).

It has also been demonstrated that the applied mechanical strain results in intracellular reactive oxygen species (ROS) accumulation and oxidative stress (OS) in pelvic support uterosacral ligament (USL) fibroblasts (17). OS influences proliferation, differentiation, apoptosis and senescence in fibroblasts (18). In addition, OS in pelvic supports increases the development of POP. Kim et al (19) identified that OS markers, 8-hydroxy-2'-deoxyguanosine (8-OHdG) and 4-hydroxy-2-nonenal (4-HNE) were increased in the USLs of patients with POP. The expression of glutathione peroxidase 1 (GPX1) in USLs of POP patients has been demonstrated to be markedly suppressed (9). Thus, the present study hypothesized that mechanical force may induce OS in pelvic supports and may be involved in the pathogenesis of POP.

Activated Akt directly phosphorylates its downstream transcription factors, which regulate expression of genes via binding with DNA. The forkhead box O (FOXO) family is an important downstream target of Akt and the phosphorylation of FOXO1 may be controlled by activated Akt, which results in nuclear exclusion and degradation, as well as inhibition of transcriptional activation. FOXO1 is involved in the control of gene transcription, for example, it decreases the expression of antioxidase (20-23), which decreases the ability of ROS detoxification and results in OS.

The present study aimed to determine the effects of mechanical loading on human USL fibroblast (hUSLF) apoptosis, senescence and production of collagen. Based on our previous studies $(17,24)$, the present study focused on the involvement of the PI3K/Akt signaling pathway and OS. The results of the present study demonstrate that mechanical strain activates Akt signaling-induced OS and affects apoptosis, senescence and collagen production in hUSLF. The present study demonstrates the importance of mechanical strain in the pathogenesis of POP, in addition to the underlying molecular mechanisms.

\section{Materials and methods}

Patients and sample collection. The present study was approved by the ethics committee of Renmin Hospital of Wuhan University was obtained prior to the commencement of the study, and written informed consent was obtained from all donors prior to sample collection. All donors underwent hysterectomy for benign indications. One year of amenorrhea in women aged $>45$ years was defined as menopause. Prior to surgery, a pelvic examination was performed to evaluate for the presence of POP. Uterovaginal prolapse was graded according to the POP quantification system advocated by the International Continence Society. Of the 56 women who underwent hysterectomy, the 20 who were diagnosed with stage II POP or greater were assigned to the POP group and the 36 without POP were assigned to the control group. Of the control group, 16 patients without POP were used to develop primary cultures of hUSLFs. Donors who had pelvic operations, pelvic inflammation, serious systemic diseases, reproductive system cancer, pelvic radiation exposure or were taking hormone replacement therapy were excluded.
Cell culture. Specimens were taken from uterosacral ligaments and fibroblasts were cultured and purified as described previously (25). Briefly, the USL tissues were cut into pieces, placed in culture bottles and digested with modified collagenase type I (Invitrogen; Thermo Fisher Scientific, Inc., Waltham, MA, USA) and trypsinase (Sigma-Aldrich, St. Louis, MO, USA). The fibroblasts were grown in serum-free Dulbecco's modified Eagle's medium (DMEM; Hyclone; GE Healthcare Life Sciences, Logan, UT, USA) supplemented with $10 \%$ fetal bovine serum (Hyclone; GE Healthcare Life Sciences), $100 \mathrm{U} / \mathrm{ml}$ penicillin/streptomycin (Beyotime Institute of Biotechnology, Haimen, China) at $37^{\circ} \mathrm{C}$ in a humidified incubator (Heal Force Development, Ltd., Hong Kong, China) with $5 \% \mathrm{CO}_{2}$. Cells were passaged at $85 \%$ confluency. The cells were characterized by their spindle-like morphology, and identified by hematoxylin and eosin staining and immunohistochemistry, which indicated positive staining for vimentin and negative staining for keratin, as previously described (17). Cells from passage 3-6 were used in the current study. Cells from 20 non-POP donors were used in the present study and each experiment was repeated in cells from at least three donors. The PI3K/Akt specific inhibitor LY294002 $(20 \mu \mathrm{M})$ dissolved in dimethyl sulfoxide (Selleck Chemicals, Houston, TX, USA) was added to cell culture $30 \mathrm{~min}$ prior to the application of mechanical strain.

Mechanical strain application. hUSLFs were seeded on flexible and transparent plates, which were pre-coated with rat tail collagen type I $(25 \mu \mathrm{g} / \mathrm{ml}$ in $0.02 \mathrm{~N}$ acetic acid; Sigma-Aldrich, St. Louis, MO, USA). Following reaching confluence, cells were rendered quiescent by incubation in serum-free DMEM for $24 \mathrm{~h}$ and prepared for mechanical strain. To exert mechanical strain on the cells, a four-point bending device (SXG4201; Chengdu Miracle Chemicals Co., Ltd., Chengdu, China) was used (17). In brief, the device was composed of a drive-control unit, loading unit and strain plates and dishes. Mechanical strain was set at $0.3 \mathrm{~Hz}$ of $5,333 \mu \varepsilon$ (loading displacement is $4 \mathrm{~mm}$ ) for the indicated time periods. When the control group cells reached confluency, the serum-free DMEM was replaced.

Western blotting. Total protein was extracted from patient tissues using radioimmunoprecipitation lysis buffer (Beyotime Institute of Biotechnology) and quantified using the BCA Protein assay kit (Beyotime Institute of Biotechnology) according to the manufacturer's protocols. Protein samples $(40 \mu \mathrm{g})$ were separated by $10 \%$ SDS-PAGE for $90 \mathrm{~min}$ at $100 \mathrm{~V}$, and transferred onto a polyvinylidene difluoride membrane, which was blocked in $5 \mathrm{~g} / 1$ skimmed milk for $1 \mathrm{~h}$. The membrane was incubated with appropriate monoclonal antibodies at $4^{\circ} \mathrm{C}$ overnight. Following washing in Tris-buffered saline with Tween 20 (TBST; Wuhan Goodbio Technology Co., Ltd., Wuhan, China), the membrane was incubated with horseradish peroxidase-conjugated goat anti-mouse (1:500; A0216) and goat anti-rabbit (1:500; A0208) secondary antibodies (Beyotime Institute of Biotechnology) at $37^{\circ} \mathrm{C}$ for $1 \mathrm{~h}$, and the target proteins were visualized using an ECL detection kit (Beyotime Institute of Biotechnology). The following primary antibodies were used: Rabbit polyclonal Akt (1:500; cat. no. 9272), rabbit monoclonal phosphorylated (p)-Akt (1:500; cat. no. 4058), mouse monoclonal FOXO1 (1:1,000; 
cat. no. 97635) and rabbit polyclonal p-FOXO1 (1:1,000; cat. no. 9461) all obtained from Cell Signaling Technology, Inc. (Danvers, MA, USA); mouse monoclonal Mn-superoxide dismutase (Mn-SOD; 1:500; cat. no. sc-130345) and rabbit polyclonal procollagen type I $\alpha 1$ (COL1A1; 1:1,000; cat. no. sc-28657) all obtained from Santa Cruz Biotechnology, Inc. (Dallas, TX, USA); and rabbit polyclonal GPX1 (1:1,000; cat. no. ab22604) and rabbit polyclonal GAPDH (1:1,000; cat. no. ab9485) obtained from Abcam, Cambridge, UK). GAPDH served as a control in the immunoblot analysis to verify the specificity of the antibody. Images of the blots were captured using a Gel-Doc XR imaging system (Bio-Rad Laboratories, Inc., Hercules, CA, USA) and they were analyzed using Quantity One 4.62 software (Bio-Rad Laboratories, Inc.).

Reverse transcription-quantitative polymerase chain reaction (RT-qPCR). Gene expression levels of GPX1, Mn-SOD, COL1A1 and GAPDH was evaluated by RT-qPCR. hUSLF RNA was extracted by using TRIzol (Invitrogen; Thermo Fisher Scientific, Inc.). DNase I (Sigma-Aldrich) was used and RNA was reverse transcribed to cDNA using a RevertAid First Strand cDNA Synthesis kit (Thermo Fisher Scientific, Inc.). The temperature protocol was as follows: $95^{\circ} \mathrm{C}$ for $30 \mathrm{sec}$; 40 cycles of $95^{\circ} \mathrm{C}$ for $5 \mathrm{sec}, 60^{\circ} \mathrm{C}$ for $34 \mathrm{sec}$, and $95^{\circ} \mathrm{C}$ for $15 \mathrm{sec} ; 60^{\circ} \mathrm{C}$ for $1 \mathrm{~min}, 95^{\circ} \mathrm{C}$ for $15 \mathrm{sec}$ and $60^{\circ} \mathrm{C}$ for $15 \mathrm{sec}$. SYBR Green I labeled probes (Takara Bio, Inc., Otsu, Japan) were used for the qPCR conducted in an ABI 7500 system (Applied Biosystems; Thermo Fisher Scientific, Inc.) with Platinum ${ }^{\circledR}$ Taq DNase (Invitrogen; Thermo Fisher Scientific, Inc.). The thermocycling conditions were as follows: $95^{\circ} \mathrm{C}$ for $30 \mathrm{sec} ; 40$ cycles of $95^{\circ} \mathrm{C}$ for $5 \mathrm{sec}$ and $60^{\circ} \mathrm{C}$ for $34 \mathrm{sec} ; 95^{\circ} \mathrm{C}$ for $15 \mathrm{sec} ; 60^{\circ} \mathrm{C}$ for $1 \mathrm{~min} ; 95^{\circ} \mathrm{C}$ for $15 \mathrm{sec}$; and $60^{\circ} \mathrm{C}$ for $1 \mathrm{~min}$. Standard curves were generated to determine the copy number of mRNA in the experimental samples, and all measurements were normalized to the expression of the GAPDH gene (data not shown). The primer sequences purchased from Beijing SBS Genetech Co., Ltd. (Beijing, China) were as follows: Forward, 5'-GCACCGTCAAGGCTGAGAAC-3' and reverse, 5'-TGGTGAAGACGCCAGTGGA-3' for GAPDH; forward, 5'-CAAGACGAAGACATCCCACCAATC-3' and reverse, 5'-ACAGATCACGTCATCGCACAACA-3' for COL1A1; and forward, 5'-CGCTTCCAGAGCATTGACATC-3' and reverse 5'-CGAGGTGGTATTTTCTGTAAGATCA-3' for GPX1. Gene expression was normalized to the expression of GAPDH, a housekeeping gene, and mRNA levels were quantified using the $2^{-\Delta \Delta \mathrm{Cq}}$ method (26).

Intracellular ROS assay. Cells $\left(1 \times 10^{5} / \mathrm{ml}\right)$ were seeded into the plates with or without exposure to strain, with $10 \mu \mathrm{M}$ serum-free DMEM containing 2',7'-dihydrodichlorofluorescein diacetate $\left(\mathrm{H}_{2} \mathrm{DCF}-\mathrm{DA}\right.$; Applygen Technologies, Inc., Beijing, China) and cultured at $37^{\circ} \mathrm{C}$. Following incubation for $30 \mathrm{~min}$ the cells were washed twice with phosphate-buffered saline (PBS; Wuhan Goodbio Technology Co.,Ltd.). The plates were observed by fluorescence microscopy (IX51; Olympus Corporation, Tokyo, Japan) at a wavelength of $450 \mathrm{~nm}$.

Intracellular $8-O H d G$ assay. Cells $\left(1 \times 10^{5} / \mathrm{ml}\right)$ were plated with or without exposure to strain and fixed with formaldehyde (Wuhan Goodbio Technology Co., Ltd.). and incubated overnight at $4^{\circ} \mathrm{C}$ with mouse monoclonal antibodies against 8-OHdG (1:200; Abcam; cat. no. ab62623). The plates were washed in TBST and incubated with goat anti-mouse secondary antibodies labeled with FITC (1:1,000; Abcam; ab7064). The nuclei were stained with 4',6-diamidino2-phenylindole. The fluorescence-stained cells were observed under fluorescence microscopy (IX51).

Flow cytometry. Annexin V (Beyotime Institute of Biotechnology)/propidium iodide (PI; Beyotime Institute of Biotechnology) double staining was conducted to detect apoptosis. Suspended cells were collected in $15 \mathrm{ml}$ centrifuge tubes $\left(5 \times 10^{6}\right.$ cells/tube), and centrifuged at $200 \times \mathrm{g}$ for $5 \mathrm{~min}$. The supernatant was discarded and the cells were resuspended with PBS. Annexin V labeled with fluorescein isothiocyanate was added, which is indicated by green fluorescence, and PI was added, which is indicated by red fluorescence, and incubated for 20 min in a dark place. The proportion of Annexin $\mathrm{V}^{+} / \mathrm{PI}^{-}$ cells were visualized by flow cytometer (FACSCalibur; BD Biosciences, Franklin Lakes, NJ, USA) and analyzed with CellQuest Pro (BD Biosciences).

In situ terminal deoxyribonucleotidyl transferase-mediated dUTP nick end labeling (TUNEL) assay. The In situ Cell Death Detection kit, Fluorescein (Roche Diagnostics GmbH, Mannheim, Germany) was used to quantify apoptosis at single cell level by labeling DNA strand breaks. Paraffin-embedded USL tissue sections were dewaxed by heating at $60^{\circ} \mathrm{C}$ and washing in xylene (Sinopharm Chemical Reagent Co., Ltd., Shanghai, China) and rehydrated with a graded series of ethanol. They were incubated with a protease $\mathrm{K}$ working solution for $20 \mathrm{~min}$ at room temperature, and then incubated with permeabilisation solution for $8 \mathrm{~min}$. Slides were rinsed twice with PBS and incubated with the TUNEL reaction mixture for $60 \mathrm{~min}$ at $37^{\circ} \mathrm{C}$ in the dark. The slides were then rinsed twice with PBS and five fields of each section was observed by fluorescence microscopy (IX51).

Senescence-associated $\beta$-galactosidase (SA- $\beta$-gal) staining. The present study used a previously described method by Dimri et al (27) to test the positive percentage of activated SA- $\beta$-gal. Cells exposed to mechanical strain were fixed by $3 \%$ formaldehyde for $5 \mathrm{~min}$. In order to detect highly expressed SA- $\beta$-gal, hUSLFs were incubated with $1 \mathrm{mg} / \mathrm{ml}$ of 5-bromo-4-chloro-3-indolyl $\beta$-D-galactopyranoside (X-Gal; Wuhan Goodbio Technology Co., Ltd.) at $37^{\circ} \mathrm{C}$ and $\mathrm{pH} 6.0$ for $12 \mathrm{~h}$. Positively stained cells were blue when observed under a light microscope (CKX31, Olympus Corporation) and the proportion of positive cells was calculated.

Statistical analysis. All experimental data points are independent and data are presented as the mean \pm standard deviation. For normally distributed data, one-way analysis of variance with Tukey's post-hoc test used for multiple comparisons and Dunnett's test for comparing each group with the control group. The Mann-Whitney U test was used to compare two groups. Statistical analysis was performed with statistical software SPSS 17.0 (SPSS, Inc., Chicago, IL, USA). $\mathrm{P}<0.05$ was considered to indicate a statistically significant difference. 
A

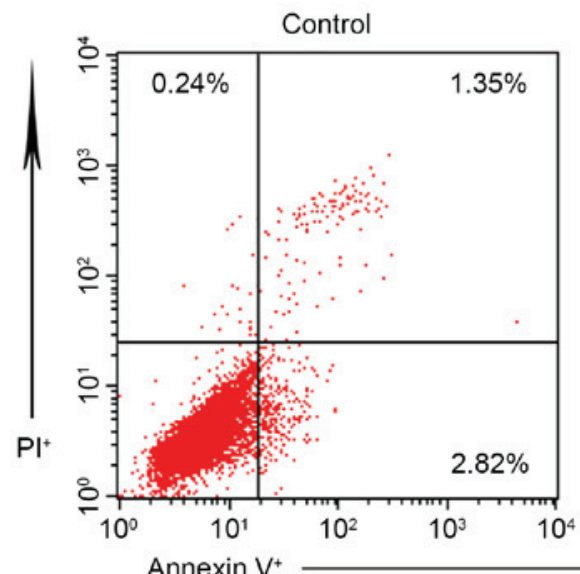

B

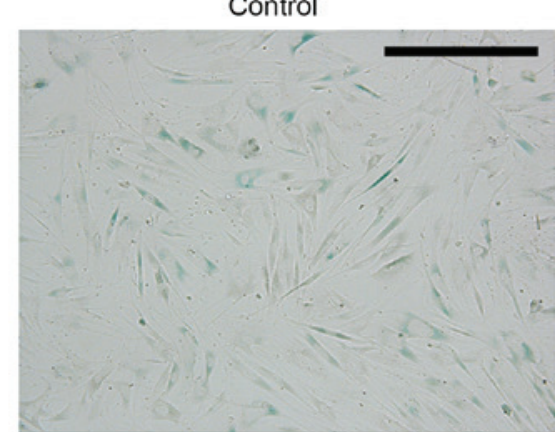

C

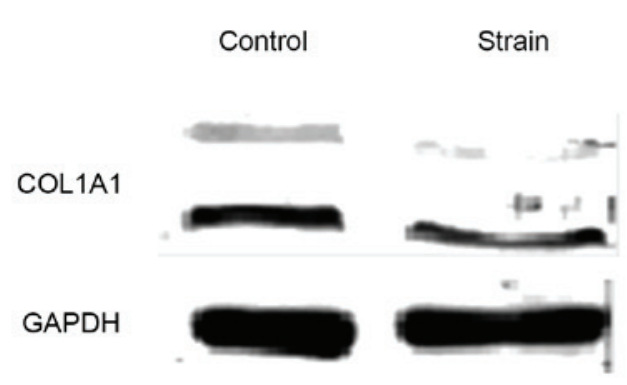

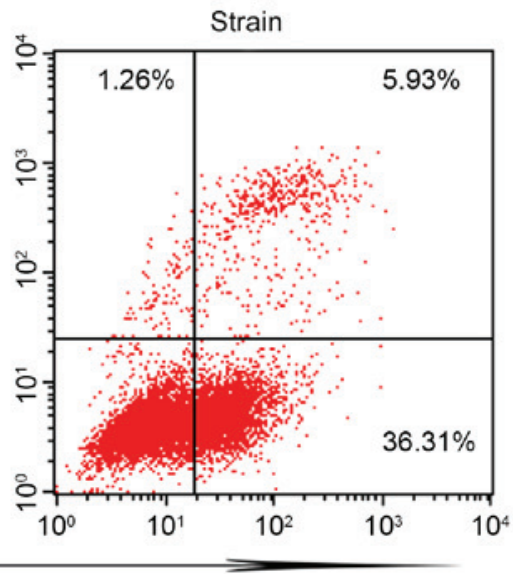

Strain

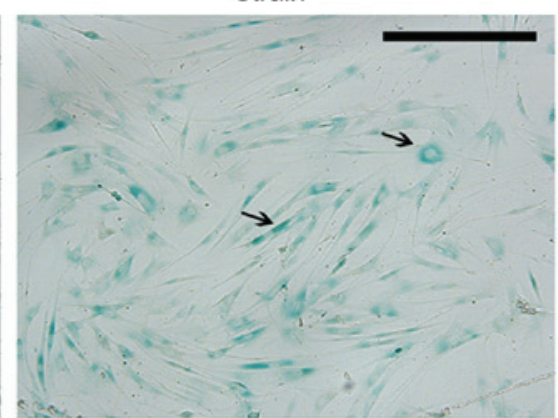

D

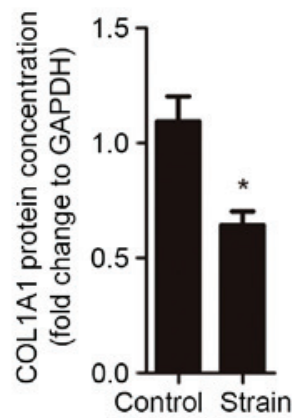

Figure 1. Mechanical strain induces hUSLF apoptosis and senescence and suppresses collagen anabolism. The hUSLF were exposed with or without mechanical strain of $5333 \mu \varepsilon$. (A) Cellular apoptosis was observed by staining with Annexin V/PI and detected by flow cytometry. (B) Cell senescence was evaluated by positive senescence-associated- $\beta$-galactosidase percentage. Scale bars, $100 \mu \mathrm{m}$ and arrows indicate examples of positive staining. (C and D) The expression of COL1A1 were assessed by western blotting. The level of COL1A1 protein concentration was calculated and normalized to the level of GAPDH protein concentration. All experiments were performed in triplicate. ${ }^{*} \mathrm{P}<0.05$ vs. the control group. hUSLF, human uterosacral ligament fibroblasts; PI, propidium iodide; COL1A1, procollagen type $1 \alpha 1$.

\section{Results}

Mechanical strain induces apoptosis and senescence and the reduces the production of collagen in hUSLFs. hUSLFs from passage 3-6 were plated on rat tail collagen type I-coated dishes until they reached confluency. A mechanical strain of $5333 \mu \varepsilon$ at $0.3 \mathrm{~Hz}$ was applied for $4 \mathrm{~h}$. In our previous experiment (24), mechanical strains of 0 , $1333 \mu \varepsilon, 2666 \mu \varepsilon$ and $5333 \mu \varepsilon$ were applied to hUSLFs for $4 \mathrm{~h}$ and Annexin V/PI double staining was used to detect cellular apoptosis. Investigation indicated that mechanical strain induced apoptosis in hUSLFs and the apoptotic percentage (Annexin $\mathrm{V}^{+} / \mathrm{PI}^{-}$) in cells exposed to $5333 \mu \varepsilon$ was markedly higher compared with other groups $(0,1333 \mu \varepsilon$ and $2666 \mu \varepsilon)(23)$. Apoptotic cells were notably higher in the group exposed to mechanical loading (Fig. 1A). Senescent cells, which are SA- $\beta$-gal positive, were also markedly increased in the mechanical loading group (Fig. 1B). It has thus been hypothesized that mechanical strain induces apoptosis and senescence in hUSLFs. Collagen is the predominant component of ECM in USL and it is produced by fibroblasts. The results of the present study indicated that protein expression levels of COL1A1 were significantly reduced in the mechanical loading fibroblasts $(\mathrm{P}<0.05$; Fig. $1 \mathrm{C}$ and $\mathrm{D})$, which indicates that mechanical strain disrupts collagen metabolism in hUSLFs. 


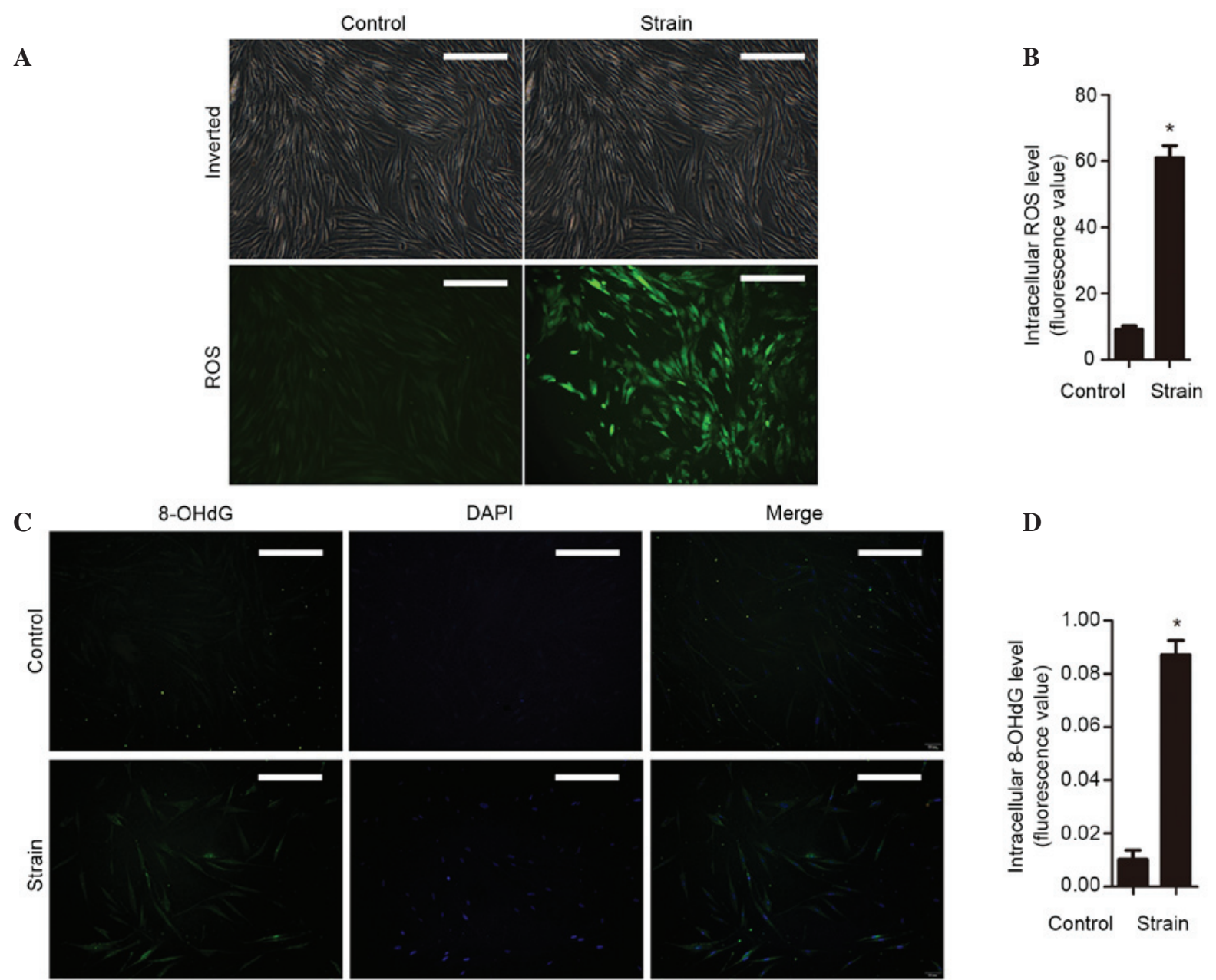

Figure 2. Mechanical strain induces oxidative stress in hUSL fibroblasts. The levels of intracellular ROS were detected by $\mathrm{H}_{2}$ DCFDA fluorescence probe. (A and B) hUSLF were seeded on the rat tail collagen type I-coated plates and loaded with strain $(5333 \mu \varepsilon$ at $0.3 \mathrm{~Hz}$ for $4 \mathrm{~h})$ following reaching confluency. Prior to the ROS assay, fibroblast dishes were observed for cellular morphology and arrangement under phase contrast microscopy. Fibroblasts were incubated with $\mathrm{H}_{2}$ DCFDA which becomes DCF indicated by green fluorescence. (C and D) Fibroblasts were fixed on the dishes and incubated with 8-OHdG antibodies and then incubated with secondary antibodies conjugated to a fluorescent dye. Cells were observed under the fluorescence microscope. 8-OHdG stained green (left), nuclei stained blue (middle) and the two images were then merged (right). Scale bars, $200 \mu \mathrm{m}$. All experiments were performed in triplicates. "P<0.05 vs. the control group. hUSLF, human uterosacral ligament fibroblasts; ROS, reactive oxygen species; DAPI, 4',6-diamidino-2-phenylindole; 8-OHdG, 8-hydroxy-2'-deoxyguanosine.

Mechanical strain increases intracellular ROS and OS. Increased intracellular ROS levels promote cellular apoptosis and senescence, and may affect cell metabolism $(28,29)$. In order to ascertain the effects of mechanical strain on intracellular ROS levels, the level of ROS was detected by fluorescent probe $\mathrm{H}_{2}$ DCFDA. The results suggested that the level of intracellular ROS were significantly increased by mechanical stress of $5333 \mu \varepsilon$ for $4 \mathrm{~h}(\mathrm{P}<0.05$; Fig. $2 \mathrm{~A}$ and B). The levels of 8 -OHdG in hUSLFs were then determined. Fibroblasts loaded with strain were observed to have significantly increased levels of 8-OHdG compared with unstimulated cells $(\mathrm{P}<0.05$; Fig. 2C and D). These results indicates that mechanical strain results in excessive accumulation of intracellular ROS and leads to OS in hUSLFs.

The PI3K/Akt signaling pathway is activated by mechanical strain in hUSLFs. Mechanical strain activates the PI3K/Akt signaling pathway and extracellular mechanical cues are transduced into intracellular signaling in order to regulate a range of effects, including apoptosis and senescence $(20,21,23)$. The transcription factor FOXO1 is phosphorylated by activated Akt and regulates expression of numerous genes, including antioxidase genes that regulate OS (20). In order to ascertain whether mechanical strain activates the PI3K/Akt signaling pathway in hUSLFs, mechanical strain of $5333 \mu \varepsilon$ at $0.3 \mathrm{~Hz}$ was applied to fibroblasts (Fig. 3). Akt was activated within $10 \mathrm{~min}$ and remained so for $1 \mathrm{~h}$, and p-Akt levels returned to basal levels in $3 \mathrm{~h}(\mathrm{P}<0.01$ at $10-120$ min; Fig. $3 \mathrm{~A}$ and $\mathrm{B})$. FOXO1 was phosphorylated for $30 \mathrm{~min}$ following exposure to mechanical strain, and returned to basal levels within $4 \mathrm{~h}$ ( $\mathrm{P}<0.001$ at 30-180 min; Fig. 3A and C). In order to investigate the association between the phosphorylation of FOXO1 and mechanical strain-activated Akt, hUSLFs were incubated with the PI3K/Akt signaling inhibitor LY294002 $(20 \mu \mathrm{M})$ for $30 \mathrm{~min}$ prior to exposure to mechanical strain. It was observed that Akt phosphorylation was blocked and FOXO1 phosphorylation was significantly inhibited $(\mathrm{P}<0.05$; Fig. $3 \mathrm{D}$ and $\mathrm{F})$. These results indicate that mechanical strain activated the PI3K/Akt signaling pathway, which in turn phosphorylates downstream FOXO1, thus resulting in its inactivation. 
A

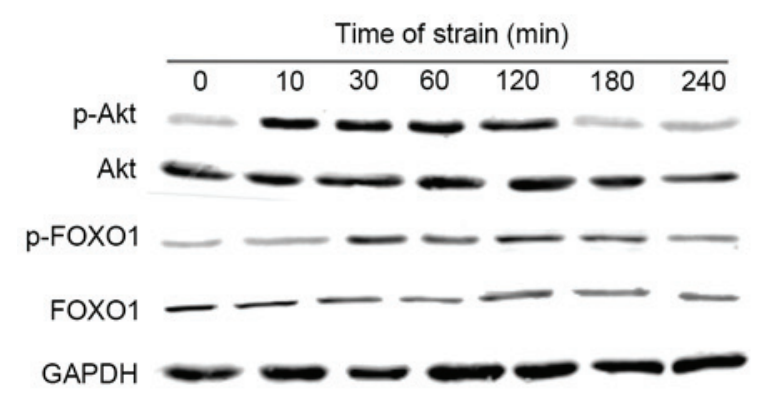

D

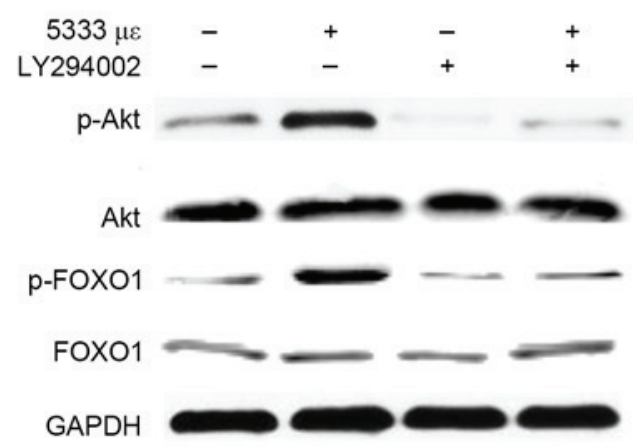

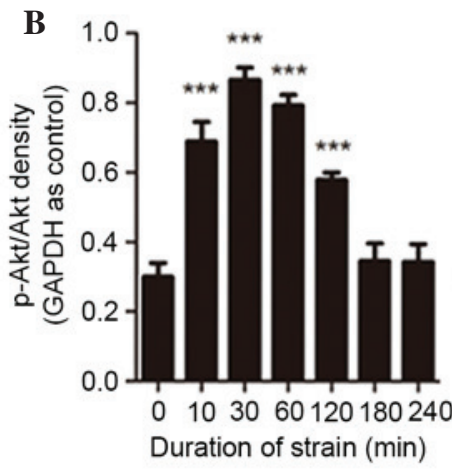

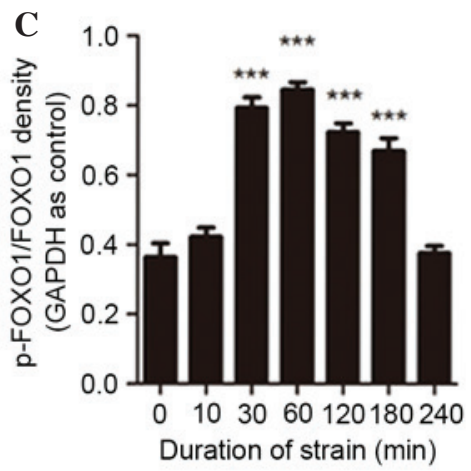

E

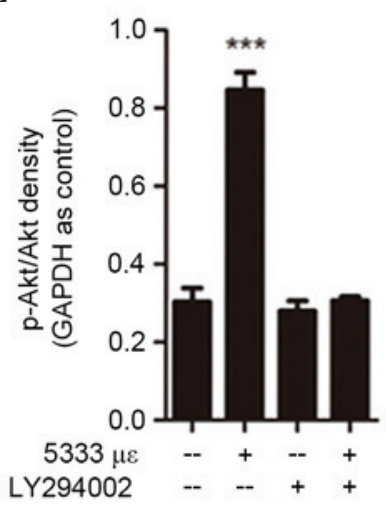

F

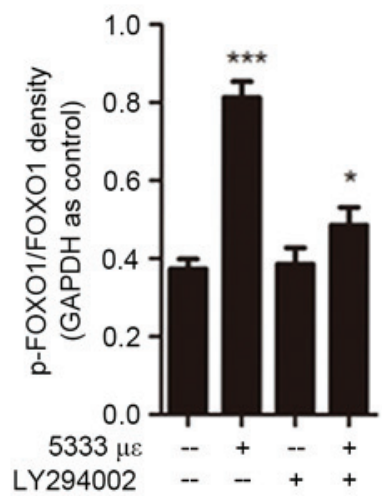

Figure 3. Mechanical strain activates the PI3K/Akt signaling pathway and inactivates downstream FOXO1. (A-C) hUSLF were seeded on rat tail collagen type I-coated plates and subjected to mechanical strain for the indicated time intervals. Akt and FOXO1 phosphorylation in hUSLF were analysed by western blotting. Unstrained cells served as the control. ${ }^{* * *} \mathrm{P}<0.001$ vs. 0 min $(\mathrm{D}-\mathrm{F})$ The protein expression levels of Akt, p-Akt, FOXO1 and p-FOXO1 in loaded cells ( $5333 \mu \varepsilon$ for $30 \mathrm{~min}$ at $0.3 \mathrm{~Hz}$ ) with or without Akt inhibitor LY294002 were detected by western blotting. GAPDH served as an internal control. "P<0.05, ${ }^{* * * *} \mathrm{P}<0.001$ vs. the other groups. Data were repeated in triplicate. PI3K, phosphatidylinositol-4,5-bisphosphate 3-kinase; FOXO1, forkhead box protein O1; hUSLF, human uterosacral ligament fibroblasts; p, phosphorylated.

A

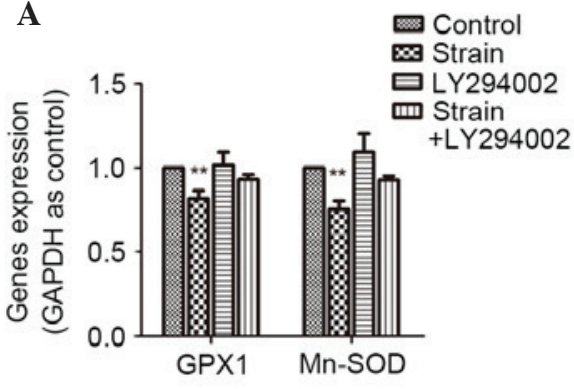

D $5333 \mu \varepsilon$

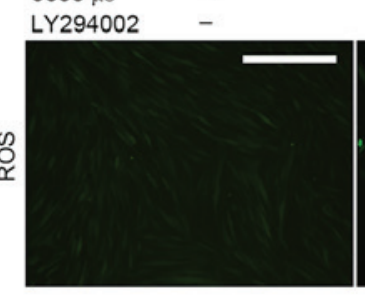

B

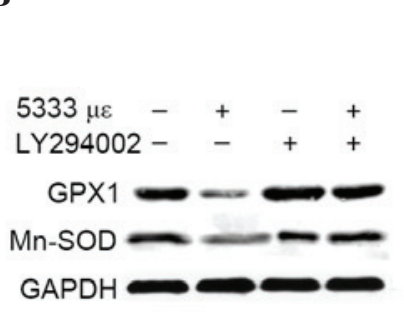

$\mathbf{C}$

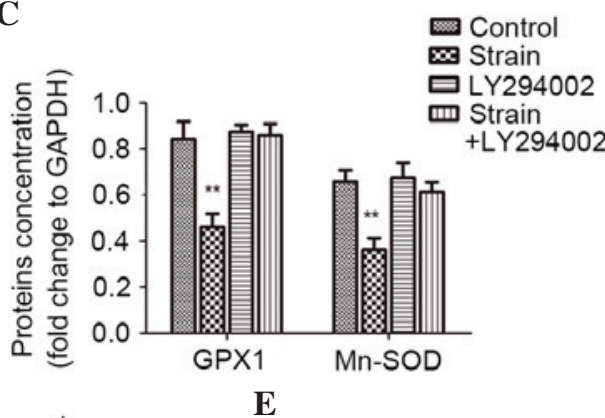

E

Figure 4. Mechanical strain induces oxidative stress by downregulation of GPX1 and Mn-SOD expression via activating PI3K/Akt signaling pathway. hUSLF were subjected to mechanical strain of $5333 \mu \varepsilon$ at $0.3 \mathrm{~Hz}$ for $4 \mathrm{~h}$ in the presence or absence of LY294002 $(20 \mu \mathrm{M}) 30$ min prior to the application of mechanical strain, and mRNA and proteins were collected. (A) GPX1 and Mn-SOD mRNA expression were assessed by reverse transcription-quantitative polymerase chain reaction. Data were calculated and normalized to GAPDH mRNA expression level. (B and C) GPX1 and Mn-SOD protein concentrations were evaluated by western blotting. GAPDH served as an internal control. Intracellular ROS levels were detected using a $\mathrm{H}_{2} \mathrm{DCFDA}$ fluorescence probe. ** $<<0.01$ vs. the control group. (D and E) hUSLF were plated on the rat tail collagen type I-coated dishes and loaded following reaching confluency. Fibroblasts were incubated with $\mathrm{H}_{2}$ DCFDA which turn into DCF as indicated by the green fluorescence. Scale bars, $100 \mu \mathrm{m}$. All experiments were performed in triplicate. "P $<0.05$, ${ }_{* * * *} \mathrm{P}<0.001$ with comparisons demonstrated part E. hUSLF, human uterosacral ligament fibroblasts; PI3K, phosphatidylinositol-4,5-bisphosphate 3-kinase; GPX1, glutathione peroxidase 1; Mn-SOD, manganese superoxide dismutase; ROS, reactive oxygen species. 

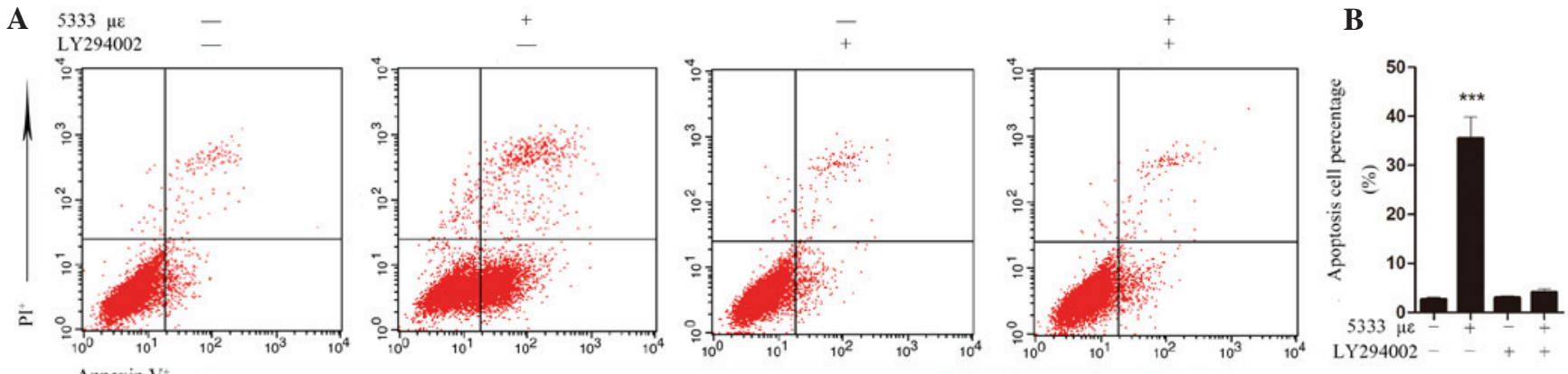

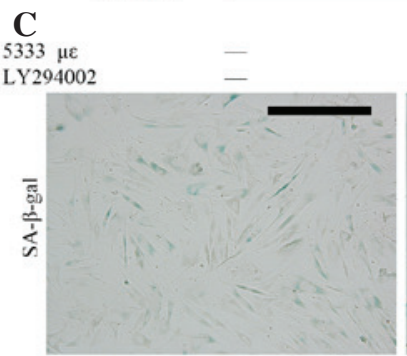

E

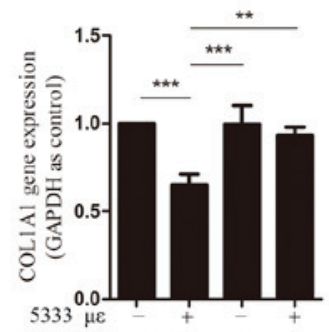

$+$

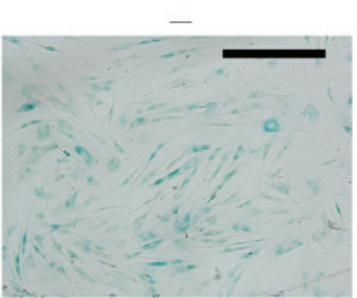

F
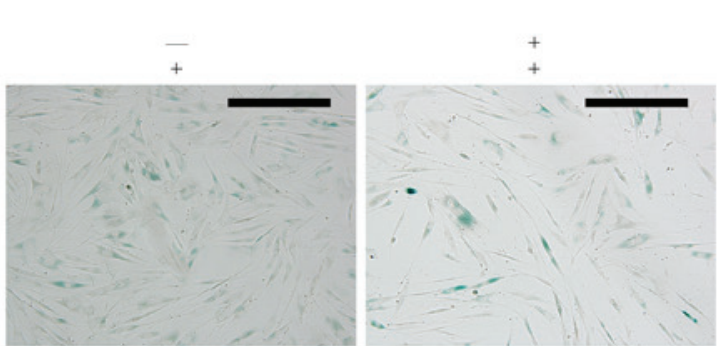

D

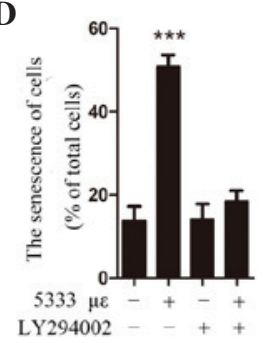

LY294002

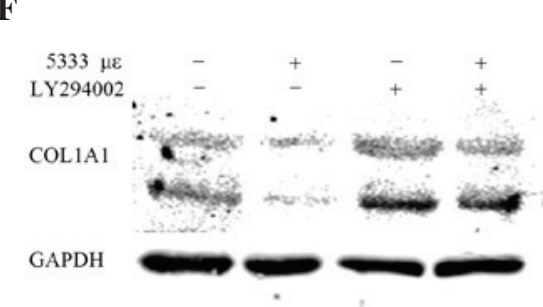

G

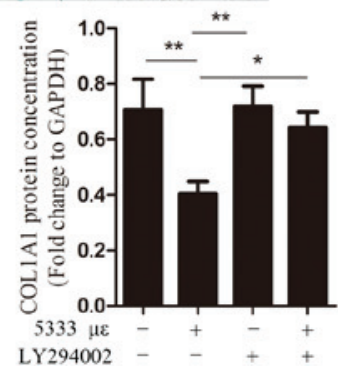

Figure 5. Mechanical strain induces hUSLF apoptosis and senescence and suppresses collagen I production via the PI3K/Akt signaling pathway. The hUSLF were treated with or without mechanical strain of $5333 \mu \varepsilon$ for $4 \mathrm{~h}$ in the presence or absence of LY294002. Cells were incubated with LY294002 (20 $\mu \mathrm{M})$ for $30 \mathrm{~min}$ prior to the application of mechanical strain. (A and B) Cellular apoptosis was detected by Annexin V/PI double staining and flow cytometer. (C and D) Cell senescence was evaluated by positive SA- $\beta$-gal percentage. Scale bars, $100 \mu \mathrm{m}$. (E-G) The mRNA and protein expression levels of COL1A1 were assessed by reverse transcription-quantitative polymerase chain reaction and western blotting. The level of COL1A1 mRNA and protein expression were calculated and normalized to the level of GAPDH. All experiments were performed in triplicate. ${ }^{*} \mathrm{P}<0.05,{ }^{* * *} \mathrm{P}<0.01,{ }^{* * *} \mathrm{P}<0.001$ vs. other groups or groups indicated. hUSLF, human uterosacral ligament fibroblasts; PI3K, phosphatidylinositol-4,5-bisphosphate 3-kinase; PI, propidium iodide; COL1A1, procollagen type $1 \alpha 1$.

Mechanical strain affects collagen anabolism via activated PI3K/Akt signaling-induced OS. The present study also examined the effect of mechanical strain on hUSLF via the PI3K/Akt/FOXO1 signaling pathway. FOXO1 regulates OS through associated gene expression. The present study demonstrated that the mRNA $(\mathrm{P}<0.01$; Fig. $4 \mathrm{~A})$ and protein $(\mathrm{P}<0.01$; Fig. 4B and C) expression levels of the antioxidases GPX1 and $\mathrm{Mn}-\mathrm{SOD}$ in fibroblasts were significantly decreased following loading with $5333 \mu \varepsilon$ strain for $4 \mathrm{~h}$, this also resulted in an increased intracellular ROS level ( $\mathrm{P}<0.001$; Fig. 4D and $\mathrm{E})$. To further investigate the role of the PI3K/Akt/FOXO1 signaling pathway in regulating OS, hUSLFs were incubated with PI3K/Akt inhibitor LY294002 prior to exposure to mechanical strain. It was observed that the expression levels of GPX1 and Mn-SOD were significantly increased as Akt activation was blocked following loading with mechanical strain $(\mathrm{P}<0.01$; Fig. 4A-C). The levels of intracellular ROS dropped evidently $(\mathrm{P}<0.001$; Fig. 4D and $\mathrm{E})$. These results demonstrate that mechanical strain changes cellular OS via the PI3K/Akt/FOXO1 signaling pathway, which regulates the expression levels of GPX1 and Mn-SOD. The role of Akt in cellular apoptosis, senescence and metabolism was also investigated. It was observed that inhibition of PI3K/Akt signaling significantly suppressed apoptosis ( $\mathrm{P}<0.001$; Fig. $5 \mathrm{~A}$ and $\mathrm{B}$ ) and senescence $(\mathrm{P}<0.001$; Fig. $5 \mathrm{C}$ and $\mathrm{D})$ and promoted the mRNA $(\mathrm{P}<0.01)$ and protein expression levels of COL1A1 $(\mathrm{P}<0.05$; Fig. 5E-G). These results indicate that mechanical strain induces apoptosis and senescence, and disrupts the production of collagen type I via activating PI3K/Akt/FOXO1-mediated OS.

PI3K/Akt signaling pathway is activated in USLs of POP patients. USLs were extracted from 20 patients with POP and 20 without POP in the course of hysterectomy. Age, body mass index and menopausal status exhibited no significant difference between two groups. Parity in the POP group was significantly increased compared with the control group $(\mathrm{P}<0.05$; Table I). Akt activation was detected in USL tissue samples from the two groups. p-Akt levels were shown to be significantly increased in USL tissue samples from the POP group compared with the control group $(\mathrm{P}<0.001 ;$ Fig. $6 \mathrm{~A}$ and $\mathrm{B})$. The data was also analyzed individually, in the POP group 12/20 USL tissue explants exhibited marked expression of p-Akt, and in three explants Akt phosphorylation was not notable. However, in control group, four explants exhibited moderate or strong p-Akt expression (data not shown). These results demonstrate 
Table I. Demographis of the POP group and non-POP group.

\begin{tabular}{|c|c|c|c|}
\hline Characteristic & POP group $(n=20)$ & Non-POP group $(n=20)$ & P-value \\
\hline Age (years), median (range) & $53.5(45-76)$ & $52(45-70)$ & $\mathrm{NS}^{\mathrm{a}}$ \\
\hline Body mass index $\left(\mathrm{kg} / \mathrm{m}^{2}\right)$, mean $\pm \mathrm{SD}$ & $26.615 \pm 3.478$ & $26.44 \pm 2.822$ & $\mathrm{NS}^{\mathrm{a}}$ \\
\hline Menopause status (years), median (range) & $10(1-36)$ & $8.5(1-30)$ & $\mathrm{NS}^{\mathrm{a}}$ \\
\hline Parity, mean $\pm \mathrm{SD}$ & $2.85 \pm 1.387$ & $2 \pm 1.026$ & $\mathrm{P}<0.05$ \\
\hline
\end{tabular}

${ }^{a}$ Mann-Whitney test. POP, pelvic organ prolapse; SD, standard deviation; NS, no significance.

A

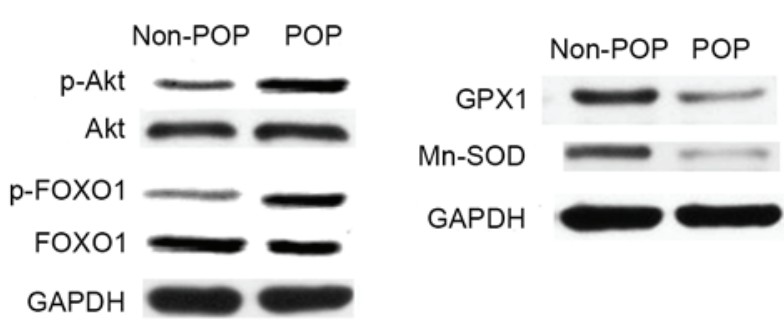

C

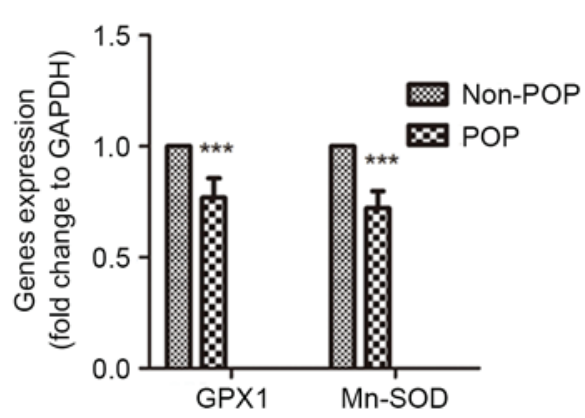

B

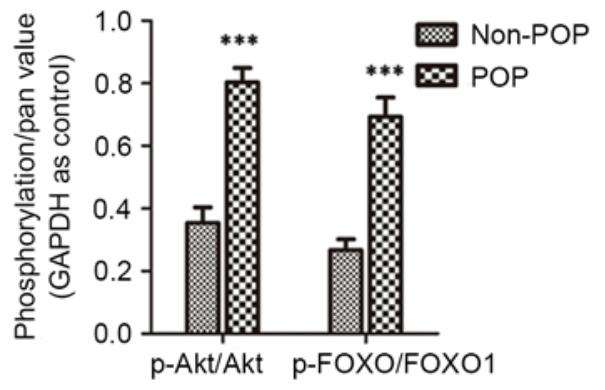

D 1.07 Non-POP

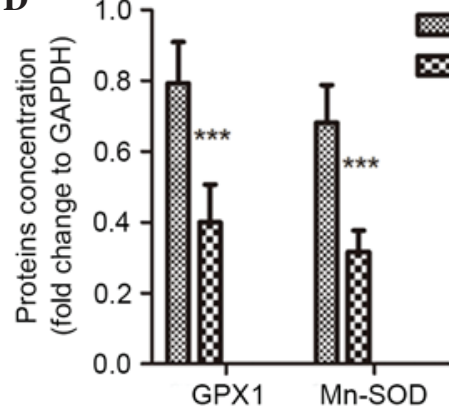

Figure 6. PI3K/Akt signaling pathway is activated and GPX1 and Mn-SOD expression were reduced in the USL of POP patients. mRNA and proteins were collected from USL tissue explants of POP and non-POP patients. The non-POP group served as a control. (A and B) Akt and p-Akt concentrations and FOXO1 and p-FOXO1 were assessed by western blotting. GAPDH served as an internal control. (C) mRNA expression of GPX1 and Mn-SOD were assessed by reverse transcription-quantitative polymerase chain reaction. Data were calculated and normalized to GAPDH mRNA expression levels. (A) GPX1 and Mn-SOD protein concentrations were detected by western blotting. (D) Data of GPX1 and Mn-SOD concentrations were calculated and normalized to GAPDH concentration. All experiments were performed in triplicate. ${ }^{* * *} \mathrm{P}<0.001$ vs. the non-POP group. USL, uterosacral ligaments; POP, pelvic organ prolapse; p, phosphorylated; FOXO1, forkhead box protein O1; GPX1, glutathione peroxidase 1; Mn-SOD, manganese superoxide dismutase.

that the PI3K/Akt signaling pathway is activated in the USLs of POP patients.

Antioxidation decreases and the production of collagen type I is reduced in USLs of POP patients. Following identification of PI3K/Akt/FOXO1 signaling pathway involvement in POP, its downstream gene targets were also investigated (Fig. 6). It was observed that mRNA $(\mathrm{P}<0.001$; Fig. 6C) and protein expression levels $(\mathrm{P}<0.001$; Fig. 6A and D) of GPX1 and Mn-SOD were significantly decreased in the POP group, which indicates a reduced defense against OS. Apoptosis in USLs was then detected by TUNEL assay, and it was observed that the percentage of TUNEL-positive cells was significantly higher in the POP group than in the control group $(\mathrm{P}<0.001$; Fig. 7A and B). The present study investigated the metabolism of collagen in pelvic supports and observed that mRNA $(\mathrm{P}<0.001)$ and protein $(\mathrm{P}<0.001)$ expression levels of COL1A1 were significantly reduced in the POP group (Fig. 7C-E).
PI3K/Akt signaling increases OS, which is involved in increased levels of cell apoptosis and reduced collagen production in pelvic supports of POP patients.

\section{Discussion}

USL, an important component of the pelvic support system, is constantly altered by exposure to the mechanical environment. Long-term high intra-abdominal pressure may result in relaxation of pelvic supports leading to POP. Fibroblasts are the predominant cell type in USLs. They transduce mechanical cues into biochemical signals to regulate expression of specific genes. In addition, hUSLF secretes ECM in response to physiological conditions. Fibroblasts are key in the maintenance of tissue homeostasis, repair and remodeling.

First, the present study investigated the mechanism of mechanical force-induced apoptosis, which is involved in the pathogenesis of POP. Clinical studies have identified that the 
A
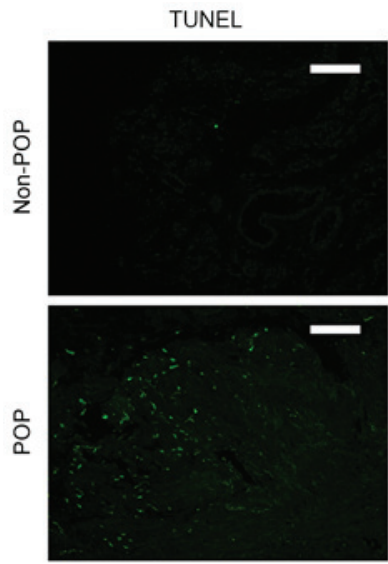

C
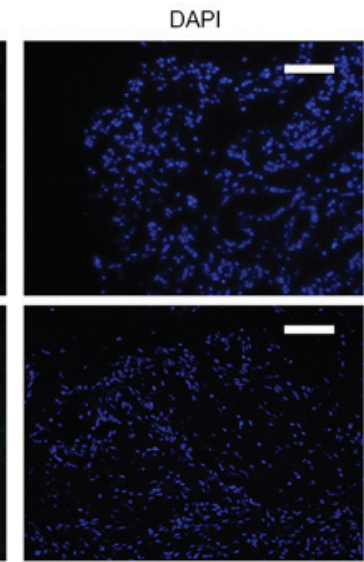

D
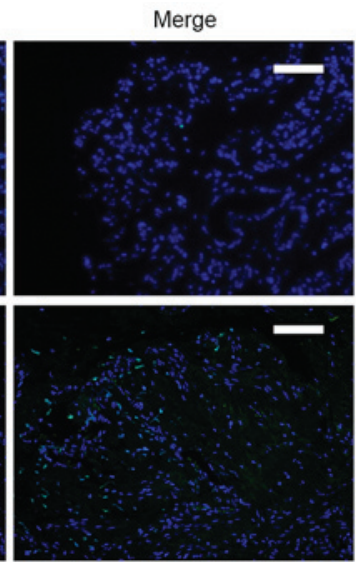

$\mathbf{E}$

B

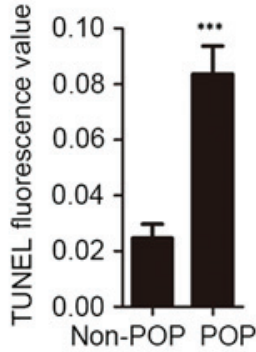

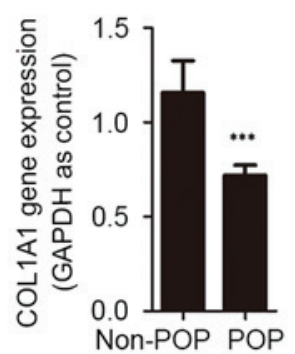
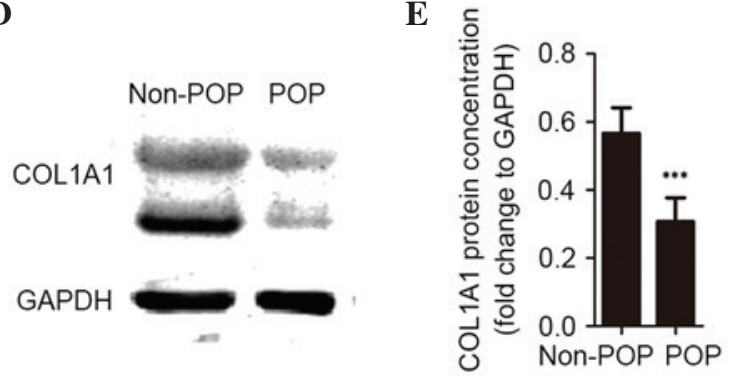

Figure 7. Apoptosis level increased and collagen type I production was reduced in USLs of POP patients. (A) Cell apoptosis in USL tissue explants were detected using the TUNEL assay. TUNEL stain positive cells stain green and nuclei were stained blue by DAPI. Scale bars, $100 \mu \mathrm{m}$. (B) Apoptosis levels were calculated by percentage of TUNEL positive cells. (C) USL RNA was collected and COL1A1 mRNA expression was detected by reverse transcription-quantitative polymerase chain reaction. GAPDH mRNA served as an internal control. (D and E) COL1A1 concentration in USL was detected by western blotting. GAPDH concentration served as an internal control. The non-POP group served as the control and all experiments performed in triplicate. ${ }^{* * *} \mathrm{P}<0.001$ vs. the non-POP group. USL, uterosacral ligament; POP, pelvic organ prolapse; TUNEL, terminal deoxynucleotidyl transferase dUTP nick end labeling; DAPI, 4',6-diamidino-2-phenylindole; COL1A1, procollagen type 1 a1.

risk factors for POP are associated with mechanical stress and that pelvic support relaxation may lead to POP. It was observed in the present study that in USLs of POP patients, cell apoptosis was increased and collagen anabolism was disrupted. In order to investigate the mechanism of POP pathogenesis in vitro, a model of hUSLFs subjected to mechanical strain was produced. A four-point bending device was used to apply mechanical stress to hUSLFs, it was observed that the stretched cells were randomly distributed and a proportion did not remain on the plates (data not shown). This mechanical strain model allowed investigation into the pathogenesis of POP. First, the effects of mechanical strain on hUSLFs were investigated and it was observed that the levels of apoptosis and senescence increased and the production of collagen type I decreased in stretch fibroblasts compared with unstretched fibroblasts. This indicated that micropathological changes in pelvic supports of POP had been imitated to a certain degree.

Excessive accumulation of intracellular ROS and OS results in cell apoptosis, senescence and abnormal metabolism (30). The present study investigated whether ROS accumulation was present in stretched cells and USLs of POP patients. The involvement of OS in the pathogenesis of POP has been previously reported (19), and the results of the present study are consistent. Mechanical strain was applied to fibroblasts and a marked increase in the level of intracellular ROS was observed, which resulted in increased OS in hUSLFs. The OS changes in the mechanically loaded hUSLF model were consistent with those in the USLs of POP patients, which verifies the validity of the model to mimic POP at micropathological levels. The $\mathrm{PI} 3 \mathrm{~K} / \mathrm{Akt}$ pathway is one of the most important signaling pathways involved in OS. Activation of PI3K/Akt is not unique to OS regulation as it also regulates normal growth and metabolism (30). However, the present study demonstrated that OS is important in apoptosis of stretched hUSLFs, and that this may be mediated by the PI3K/Akt signaling pathway.

Mechanical strain activates a number of signaling pathways, including PI3K/Akt to transduce extracellular stimulation into intracellular signals, and regulate various cellular responses. The transcription factor FOXO1, a downstream target of the PI3K/Akt signaling pathway, controls numerous genes, including antioxidase genes that protect cells against oxidative injury (22). The expression of PI3K/Akt signaling regulates cell survival and proliferation partly by phosphorylating FOXO proteins to promote their export from the nucleus and degradation via the ubiquitin proteasome pathway-dependent pathway (20). In order to elucidate the effects of mechanical strain on the PI3K/Akt signaling pathway, $5333 \mu \varepsilon$ mechanical strain at $0.3 \mathrm{~Hz}$ was applied to hUSLFs. Akt was activated rapidly and remained activated, prior to gradual inactivation. In addition, rapid Akt activation was followed by FOXO1 phosphorylation resulting in FOXO1 nuclear exclusion and a reduced ability to regulate target genes. It has been reported that transcription factor FOXO family bind to target genes in the nucleus and regulate their expression, including genes associated with OS, DNA injury, the cell cycle, apoptosis, metabolism $(20,22,23)$. In the present study, hUSLFs were 
incubated with a specific PI3K/Akt inhibitor LY294002 prior to exposure to mechanical strain. It was observed that LY294002 blocked the activation of Akt and FOXO1 phosphorylation was markedly reduced. This may be due to another upstream signaling pathway activated by mechanical stress that also regulates FOXO1. It has been reported that activation of the extracellular signal-regulated kinase $1 / 2$ or c-Jun amino-terminal kinase signaling pathways phosphorylates FOXO1 (31-33). This suggests that mechanical strain-induced FOXO1 phosphorylation is predominantly regulated by PI3K/Akt signaling activation in hUSLFs.

The results of the present study demonstrate that mechanical strain suppresses the expression of GPX1 and Mn-SOD. It also decreases the ability to scavenge ROS, which results in excessive ROS accumulation in hUSLFs. High levels of ROS in cells disrupts the normal redox balance and cells enter a state of OS. When OS is severe and cell defense is weak, cells may undergo apoptosis, and a sustained increase in ROS may function as a common trigger for activating senescence (30). Increased ROS in cells may also affect metabolism, suppress cell viability and damage gene expression or signaling transduction, which may block collagen anabolism. In order to investigate whether mechanical strain-induced OS promoted apoptosis and senescence. and interferes with collagen production, hUSLFs were incubated with LY294002 prior to the exposure to mechanical strain. It was observed that the expression levels of GPX1 and $\mathrm{Mn}-\mathrm{SOD}$ were increased and the levels of intracellular ROS were decreased compared with cells exposed to mechanical strain but not the inhibitor. In addition, the degree of the apoptosis and senescence was reduced and COL1A1 expression was increased following the inhibition of Akt. However, the levels did to return to the basal standard, which indicates there may be effects due to a small quantity of unphosphorylated FOXO1 that was not blocked by LY294002, or may be regulated by other signaling pathways. Although it is possible that the PI3KAkt/FOXO1 signaling pathway is not the only pathway involved in the effects of mechanical strain on hUSLFs, the results demonstrated that is the predominant signaling pathway.

In conclusion, the in vitro experiments in the present study demonstrated that mechanical strain induces apoptosis and senescence, and reduces collagen type I production via activated PI3K/Akt signaling pathway-mediated OS. Mechanical strain activates Akt and, thus, downstream FOXO1 is phosphorylated and the expression of GPX1 and Mn-SOD is suppressed, which results in a decreased ability to scavenge ROS. In addition, the current study observed that the PI3K/Akt signaling pathway is activated and antioxidation declined in USLs of POP patients. The present study indicates that mechanical strain results in PI3K/Akt-mediated OS, which is key in the pathogenesis of POP and may have potential as a therapeutic strategy for POP.

\section{Acknowledgements}

The authors would like to thank their colleagues in the Department of Obstetrics and Gynecology, Renmin Hospital of Wuhan University for helping extract uterosacral ligament explants in the course of hysterectomy surgeries. The present study was funded by the National Natural Science Foundation of China (grant no. 81270684 and 81471442).

\section{References}

1. Jelovsek JE, Maher C and Barber MD: Pelvic organ prolapse. Lancet 369: 1027-1038, 2007.

2. Virtanen HS and Mäkinen JI: Retrospective analysis of 711 patients operated on for pelvic relaxation in 1983-1989. Int J Gynaecol Obstet 42: 109-115, 1993.

3. Gyhagen M, Bullarbo M, Nielsen TF and Milsom I: Prevalence and risk factors for pelvic organ prolapse 20 years after childbirth: A national cohort study in singleton primiparae after vaginal or caesarean delivery. BJOG 120: 152-160, 2013.

4. Chow D and Rodríguez LV Epidemiology and prevalence of pelvic organ prolapse. Curr Opin Urol 23: 293-298, 2013.

5. Miedel A, Tegerstedt G, Mæhle-Schmidt M, Nyrén O and Hammarström M: Nonobstetric risk factors for symptomatic pelvic organ prolapse. Obstet Gynecol 113: 1089-1097, 2009.

6. Paterno J, Vial IN, Wong VW, Rustad KC, Sorkin M, Shi Y, Bhatt KA, Thangarajah H, Glotzbach JP and Gurtner GC: Akt-mediated mechanotransduction in murine fibroblasts during hypertrophic scar formation. Wound Repair Regen 19: 49-58, 2011.

7. Zhou Y, Ling O and Bo L: Expression and significance of lysyl oxidase-like 1 and fibulin-5 in the cardinal ligament tissue of patients with pelvic floor dysfunction. J Biomed Res 27: 23-28, 2013.

8. Budatha M, Silva S, Montoya TI, Suzuki A, Shah-Simpson S, Wieslander CK, Yanagisawa M, Word RA and Yanagisawa H: Dysregulation of protease and protease inhibitors in a mouse model of human pelvic organ prolapse. PloS One 8: e56376, 2013.

9. Li BS, Hong L, Min J, Wu DB, Hu M and Guo WJ: The expression of glutathione peroxidase- 1 and the anabolism of collagen regulation pathway transforming growth factor-betal-connective tissue growth factor in women with uterine prolapse and the clinic significance. Clin Exp Obstet Gynecol 40: 586-590, 2013.

10. Konstantonis D, Papadopoulou A, Makou M, Eliades T, Basdra E and Kletsas D: The role of cellular senescence on the cyclic stretching-mediated activation of MAPK and ALP expression and activity in human periodontal ligament fibroblasts. Exp Gerontol 57: 175-180, 2014

11. Yue Y, Lypowy J, Hedhli $\mathrm{N}$ and Abdellatif $\mathrm{M}$ : Ras GTPase-activating protein binds to Akt and is required for its activation. J Biol Chem 279: 12883-12889, 2004.

12. Nho RS, Xia H, Kahm J, Kleidon J, Diebold D and Henke CA: Role of integrin-linked kinase in regulating phosphorylation of Akt and fibroblast survival in type I collagen matrices through a betal integrin viability signaling pathway. J Biol Chem 280: 26630-26639, 2005 .

13. Thompson WR, Rubin CT and Rubin J: Mechanical regulation of signaling pathways in bone. Gene 503: 179-193, 2012.

14. Xue Z, Zhang W, Desai LP, Gao H, Gunst SJ and Tepper RS: Increased mechanical strain imposed on murine lungs during ventilation in vivo depresses airway responsiveness and activation of protein kinase Akt. J Appl Physiol (1985) 114: 1506-1510, 2013.

15. Boccafoschi F, Bosetti M, Sandra PM, Leigheb M and Cannas M: Effects of mechanical stress on cell adhesion: A possible mechanism for morphological changes. Cell Adh Migr 4: 19-25, 2010.

16. Premaraj S, Souza I and Premaraj T: Mechanical loading activates $\beta$-catenin signaling in periodontal ligament cells. Angle Orthod 81: 592-599, 2011.

17. Hong SS, Ding WJ, Wu DB, Min J, Hong L, et al: Effects of oxidative damage in human parametrial ligament fibroblasts induced by mechanical stress. Chin J Clinicians (Electronic Edition) 7: 10775-10779, 2013.

18. Usta A, Guzin K, Kanter M, Ozgül M and Usta CS: Expression of matrix metalloproteinase-1 in round ligament and uterosacral ligament tissue from women with pelvic organ prolapse. J Mol Histol 45: 275-281, 2014.

19. Kim EJ, Chung N, Park SH, Lee KH, Kim SW, Kim JY, Bai SW and Jeon MJ: Involvement of oxidative stress and mitochondrial apoptosis in the pathogenesis of pelvic organ prolapse. J Urol 189: 588-594, 2013.

20. Zhang X, Tang N, Hadden TJ and Rishi AK: Akt, FoxO and regulation of apoptosis. Biochim Biophys Acta 1813: 1978-1986, 2011.

21. Fu Z and Tindall DJ: FOXOs, cancer and regulation of apoptosis. Oncogene 27: 2312-2319, 2008.

22. Downing JR: A new FOXO pathway required for leukemogenesis. Cell 146: 669-670, 2011. 
23. Tzivion $\mathrm{G}$ and Hay N: PI3K-AKT-FoxO axis in cancer and aging. Biochim Biophys Acta 1813: 1925, 2011.

24. Hong S, Li H, Wu D, Li B, Liu C, Guo W, Min J, Hu M, Zhao Y, and Yang Q: Oxidative damage to human parametrial ligament fibroblasts induced by mechanical stress. Mol Med Rep 12: 5342-5348, 2015

25. Ding WJ, Hong SS, Min J, Fang G, Zhang X, Hu M, Yang Q and Hong L: Preliminary study on primary culture of human parametrial ligament fibroblasts. Medical Journal of Wuhan University 35: 16-19, 2014.

26. Livak KJ and Schmittgen TD: Analysis of relative gene expression data using real-time quantitative PCR and the 2(-delta delta $\mathrm{C}(\mathrm{T}))$ method. Methods 25: 402-408, 2001.

27. Dimri GP, Lee X, Basile G, Acosta M, Scott G, Roskelley C, Medrano EE, Linskens M, Rubelj I, Pereira-Smith O, et al: A biomarker that identifies senescent human cells in culture and in aging skin in vivo. Proc Natl Acad Sci USA 92: 9363-9367, 1995.
28. Franklin JL: Redox regulation of the intrinsic pathway in neuronal apoptosis. Antioxid Redox Signal 14: 1437-1448, 2011.

29. Labunskyy VM and Gladyshev VN: Role of reactive oxygen species-mediated signaling in aging. Antioxid Redox Signal 19: $1362-1372,2013$

30. Finkel T and Holbrook NJ: Oxidants, oxidative stress and the biology of ageing. Nature 408: 239-247, 2000.

31. Mori R, Tanaka K, de Kerckhove M, Okamoto M, Kashiyama K, Tanaka K, Kim S, Kawata T, Komatsu T, Park S, et al: Reduced FOXO1 expression accelerates skin wound healing and attenuates scarring. Am J Pathol 184: 2465-2479, 2014.

32. Danciu TE, Gagari E, Adam RM, Damoulis PD and Freeman MR: Mechanical strain delivers anti-apoptotic and proliferative signals to gingival fibroblasts. J Dent Res 83: 596-601, 2004.

33. Chae HD and Broxmeyer HE: SIRT1 deficiency downregulates PTEN/JNK/FOXO1 pathway to block reactive oxygen species-induced apoptosis in mouse embryonic stem cells. Stem Cells Dev 20: 1277-1285, 2011. 